\title{
INDUCTION OF CHARACTERS AND FINITE $p$-GROUPS
}

\author{
EDITH ADAN-BANTE \\ University of Southern Mississippi Gulf Coast, 730 East Beach Boulevard, Long Beach MS 39560 \\ e-mail: Edith.Bante@usm.edu
}

(Received 24 February, 2006; revised 20 May, 2006; accepted 3 June, 2006)

\begin{abstract}
Let $G$ be a finite $p$-group, where $p$ is an odd prime number, $H$ a subgroup of $G$ and $\theta \in \operatorname{Irr}(H)$ an irreducible character of $H$. Assume also that $|G: H|=p^{2}$. Then the character $\theta^{G}$ of $G$ induced by $\theta$ is either a multiple of an irreducible character of $G$, or has at least $\frac{p+1}{2}$ distinct irreducible constituents.
\end{abstract}

2000 Mathematics Subject Classification. 20C15.

1. Introduction. Let $G$ be a finite group. Denote by $\operatorname{Irr}(G)$ the set of irreducible complex characters of $G$. Throughout this work, we use the notation of [2]. In addition, we are going to denote by $\operatorname{Lin}(G)=\{\lambda \in \operatorname{Irr}(G) \mid \lambda(1)=1\}$ the set of linear characters.

Let $\Gamma$ be a character of $G$. Then $\Gamma$ can be expressed as a nontrivial integral linear combination of distinct irreducible characters of $G$. Denote by $\eta(\Gamma)$ the number of distinct irreducible constituents of $\Gamma$.

Let $G$ be a finite $p$-group, where $p$ is a prime number, $H$ be a subgroup of $G$ and $\theta \in \operatorname{Irr}(H)$. Denote by $\theta^{G}$ the character of $G$ induced by $\theta$. If $H$ is a normal subgroup, then either $\eta\left(\theta^{G}\right)=1$, i.e. $\theta^{G}$ is a multiple of an irreducible, or $\eta\left(\theta^{G}\right) \geq p$, i.e. $\theta^{G}$ is an integral linear combination of at least $p$ distinct irreducible characters of $G$ (see Lemma 2.2). In Theorem 4.15, it is shown that given any prime $p>2$ and any integer $l \geq 2$, there exist a $p$-group $G$, a subgroup $H$ of $G$ with $|G: H|=p^{l}$ and $\theta \in \operatorname{Irr}(H)$ such that $\eta\left(\theta^{G}\right)=\frac{p+1}{2}$. Therefore Lemma 2.2 does not remain true without the hypothesis that $H$ is normal in $G$. But given any prime $p>2$ and any integer $n>0$, do there exist a $p$-group $G$, a subgroup $H$ of $G$ and $\theta \in \operatorname{Irr}(H)$ with $\eta\left(\theta^{G}\right)=n$ ? If we also required, in addition, that $|G: H|=p^{2}$ and $1<n<\frac{p+1}{2}$, then the answer is no. More specifically:

TheOREM A. Let $G$ be a finite p-group, where $p$ is an odd prime number, $H$ be a subgroup of $G$ and $\theta \in \operatorname{Irr}(H)$. Assume also that $|G: H|=p^{2}$. Then either $\eta\left(\theta^{G}\right)=1$ or $\eta\left(\theta^{G}\right) \geq \frac{p+1}{2}$.

For a fixed prime $p>3$, Theorem A implies that there exists a "gap" among the possible values that $\eta\left(\theta^{G}\right)$ can take for any finite $p$-group $G$, any subgroup $H$ of $G$ with $|G: H|=p^{2}$, and any character $\theta \in \operatorname{Irr}(H)$. But, do there exist a $p$-group $G$, a subgroup $H$ of $G$ and $\theta \in \operatorname{Irr}(H)$ with $1<\eta\left(\theta^{G}\right)<\frac{p+1}{2}$ and $|G: H|>p^{2}$ ? The answer is yes. In Theorem 4.23 , given any prime $p$ such that 3 divides $p-1$, we provide a p-group $G$, a subgroup $H$ of $G$ with $|G: H|=p^{3}$ and a character $\lambda \in \operatorname{Lin}(H)$ such that $\eta\left(\lambda^{G}\right)=\frac{p+2}{3}$. Does it mean then that, for a fixed prime $p>5$, there are no "gaps" among the possible values that $\eta\left(\theta^{G}\right)$ can take for any finite $p$-group $G$, any subgroup $H$ of $G$ with $|G: H|=p^{3}$, and any character $\theta \in \operatorname{Irr}(H)$ ? We do not know the answer of that question. 


\section{Preliminaries.}

LEMMA 2.1. Let $G$ be a finite group, $N$ be a normal subgroup of $G$ and $\theta \in \operatorname{Irr}(N)$. Let $G_{\theta}$ be the stabilizer of $\theta$ in $G$. Then $\eta\left(\theta^{G}\right)=\eta\left(\theta^{G_{\theta}}\right)$.

Proof. Observe that all the irreducible constituents of $\theta^{G_{\theta}}$ lie above $\theta$. Thus by Clifford theory it follows that $\eta\left(\theta^{G}\right)=\eta\left(\theta^{G_{\theta}}\right)$.

LEMMA 2.2. Let $G$ be a finite p-group, $H$ be a normal subgroup of $G$ and $\theta \in \operatorname{Irr}(H)$. Then either $\eta\left(\theta^{G}\right)=1$ or $\eta\left(\theta^{G}\right) \geq p$.

Proof. In [1, Lemma 4.1], it is proved that, if in addition to the previous hypothesis, $\theta$ is $G$-invariant, then $\eta\left(\theta^{G}\right)=1$ or $\eta\left(\theta^{G}\right) \geq p$. Thus by induction on $|G: H|$ and Lemma 2.1, the result follows.

Let $G$ be a group, $H$ be a subgroup of $G$ and $\theta \in \operatorname{Irr}(H)$. Denote by $\operatorname{Irr}(G \mid \theta)=$ $\left\{\chi \in \operatorname{Irr}(G) \mid\left[\chi_{H}, \theta\right] \neq 0\right\}$ the set of irreducible characters of $G$ lying above $\theta$.

LEMMA 2.3. Let $G$ be a finite p-group, $H$ be a subgroup of $G$ and $\theta \in \operatorname{Irr}(H)$. Let $Z_{1}$ be a subgroup of the center $\mathbf{Z}(G)$ of $G$ such that $\left|H Z_{1}: H\right|=p$. Then $\theta$ extends to $H Z_{1}$ and

$$
\eta\left(\theta^{G}\right)=\sum_{\nu \in \operatorname{Irr}\left(H Z_{1} \mid \theta\right)} \eta\left(v^{G}\right) .
$$

In particular, if $v \in \operatorname{Irr}\left(H Z_{1} \mid \theta\right)$ we have that

$$
\eta\left(\theta^{G}\right) \geq \eta\left(v^{G}\right)+(p-1) .
$$

Proof. Observe that $\theta$ extends to $H Z_{1}$ since $Z_{1} \leq \mathbf{Z}(G)$ and $\left|H Z_{1}: H\right|=p$. Thus there are exactly $p$ characters in $\operatorname{Irr}\left(H Z_{1} \mid \theta\right)$. Let $\alpha \in \operatorname{Lin}\left(H \cap Z_{1}\right)$ be the unique character such that $\theta_{H \cap Z_{1}}=\theta(1) \alpha$. Since $\left(\theta^{H Z_{1}}\right)_{Z_{1}}=\left(\theta_{H \cap Z_{1}}\right)^{Z_{1}}$, we have that $\left(\theta^{H Z_{1}}\right)_{Z_{1}}=$ $\theta(1) \sum_{v \in \operatorname{Lin}\left(Z_{1} \mid \alpha\right)} v$. Therefore

$$
\text { for any } v, \mu \in \operatorname{Irr}\left(H Z_{1} \mid \theta\right) \text {, if } v \neq \mu \text { then } v_{Z_{1}} \neq \mu_{Z_{1}} \text {. }
$$

Observe that for any $\chi \in \operatorname{Irr}(G)$ and any $\beta \in \operatorname{Lin}\left(Z_{1}\right)$, if $\left[\chi_{Z_{1}}, \beta\right] \neq 0$ then $\chi_{Z_{1}}=$ $\chi(1) \beta$. By (2.5), it follows that if $\chi, \psi \in \operatorname{Irr}(G), v, \mu \in \operatorname{Irr}\left(H Z_{1} \mid \theta\right), v \neq \mu,\left[\chi_{Z_{1}}, \nu\right] \neq 0$ and $\left[\psi_{Z_{1}}, \mu\right] \neq 0$, then $\chi \neq \psi$. Thus the irreducible constituents of $\theta^{G}$ lying over distinct extensions of $\theta$ in $H Z_{1}$ are distinct characters. It follows that

$$
\eta\left(\theta^{G}\right)=\sum_{v \in \operatorname{Irr}\left(H Z_{1} \mid \theta\right)} \eta\left(v^{G}\right)
$$

Since $\eta\left(v^{G}\right) \geq 1$ for any $v \in \operatorname{Irr}\left(H Z_{1}\right),(2.4)$ follows.

3. Proof of Theorem A. Let $G$ and $\theta \in \operatorname{Irr}(H)$ be a minimal counterexample of the statement of Theorem A with respect to the order $|G|$ of $G$. That is we are assuming that

$$
|G: H|=p^{2}, 1<\eta\left(\theta^{G}\right)<\frac{p+1}{2}
$$


and for any finite p-group $G_{1}$, any subgroup $H_{1}$ of $G_{1}$, and any $\theta_{1} \in \operatorname{Irr}\left(H_{1}\right)$, if

$$
\left|G_{1}: H_{1}\right|=p^{2} \text { and }\left|G_{1}\right|<|G| \text { then either } \eta\left(\theta_{1}^{G_{1}}\right)=1 \text { or } \eta\left(\theta_{1}^{G_{1}}\right) \geq \frac{p+1}{2} \text {. }
$$

Set $\bar{L}=L / \operatorname{core}_{G}(\operatorname{Ker}(\theta))$ for any subgroup $L$ of $G$ such that $L \geq \operatorname{core}_{G}(\operatorname{Ker}(\theta))$. Observe that $H \geq \operatorname{core}_{G}(\operatorname{Ker}(\theta))$ and $|\bar{G}: \bar{H}|=|G: H|$. Observe also that we can regard $\theta$ as a character of $H / \operatorname{core}_{G}(\operatorname{Ker}(\theta))$ and $\eta\left(\theta^{\bar{G}}\right)=\eta\left(\theta^{G}\right)$.

By working with the group $G / \operatorname{core}_{G}(\operatorname{Ker}(\theta))$ and (3.2), we may assume that

$$
\operatorname{core}_{G}(\operatorname{Ker}(\theta))=1 .
$$

Thus $\bar{L}=L$ for all subgroups $L$ of $G$.

Denote by $Z$ the center $\mathbf{Z}(G)$ of $G$.

Claim 3.3. $Z<H$. Let $v \in \operatorname{Lin}(Z)$ be the unique character of $Z$ lying below $\theta$. Then $v \in \operatorname{Lin}(Z)$ is a faithful character of $Z$ and $Z$ is a cyclic group.

Proof. Suppose $Z$ is not contained in $H$. Let $Z_{1} \leq Z$ be such that $\left|H Z_{1}: H\right|=p$. Lemma 2.3 implies that $\eta\left(\theta^{G}\right) \geq p$, a contradiction with (3.1). Thus $Z \leq H$. Since $Z=H$ implies that $H$ is normal, by Lemma 2.2 we must have that $Z<H$.

Since $\operatorname{Ker}(\theta) \cap Z$ is normal in $G$ and $\operatorname{core}_{G}(\operatorname{Ker}(\theta))=1$, it follows that $\theta_{Z}$ is a faithful character of $Z$. Therefore $v \in \operatorname{Lin}(Z)$ is faithful and $Z$ is cyclic.

Claim 3.4. $\operatorname{core}_{G}(H)=Z$.

Proof. Assume that there exists a normal subgroup $N$ of $G$ such that $N \leq H$ and $N / Z$ is a chief factor of $G$. Fix $\beta \in \operatorname{Irr}(N)$ such that $\left[\theta_{N}, \beta\right] \neq 0$. Since $v \in \operatorname{Lin}(Z)$ is a faithful character, we can check that $\mathbf{C}_{G}(N)$ is a normal subgroup of $G$ of index $p$. Also the stabilizer $G_{\beta}$ of $\beta$ in $G$ is $\mathbf{C}_{G}(N)$.

If $H \cap \mathbf{C}_{G}(N)<H$, by Clifford theory we have that there exists some $\alpha \in \operatorname{Irr}(H \cap$ $\left.\mathbf{C}_{G}(N)\right)$ such that $\alpha^{H}=\theta$. Thus $\eta\left(\theta^{G}\right)=\eta\left(\alpha^{G}\right)$. Since $\left|\mathbf{C}_{G}(N)\right|<|G|$ and $\mid \mathbf{C}_{G}(N): H \cap$ $\mathbf{C}_{G}(N) \mid=p^{2}$, by (3.2) we have that $\eta\left(\alpha^{\mathbf{C}_{G}(N)}\right)=1$ or $\eta\left(\alpha^{\mathbf{C}_{G}(N)}\right) \geq \frac{p+1}{2}$. By Lemma 2.1 we have then that $\eta\left(\alpha^{G}\right)=1$ or $\eta\left(\alpha^{G}\right) \geq \frac{p+1}{2}$ and therefore $\eta\left(\theta^{G}\right)=1$ or $\eta\left(\theta^{G}\right) \geq \frac{p+1}{2}$, a contradiction with (3.1). We may assume then that $H<\mathbf{C}_{G}(N)$.

Since $\left|\mathbf{C}_{G}(N): H\right|=p, H$ is normal in $\mathbf{C}_{G}(N)$ and thus by Lemma 2.2 we have that either $\eta\left(\theta^{\mathbf{C}_{G}(N)}\right)=1$ or $\eta\left(\theta^{\mathbf{C}_{G}(N)}\right)=p$. By Lemma 2.1 and the previous statement, we have that $\eta\left(\theta^{G}\right)=1$ or $\eta\left(\theta^{G}\right) \geq p$, a contradiction with (3.1). Thus such $N$ cannot exist and so $\operatorname{core}_{G}(H)=Z$.

Let $Y / Z$ be a chief factor of $G$. By the previous claim, it follows that $H Y>H$. Since $Y / Z$ has order $p$, we have that $|H Y: H|=p$. Since $|G: H|=p^{2}$, it follows that $|G: H Y|=p$ and thus $H Y$ is a normal subgroup of $G$.

Set $C=\mathbf{C}_{G}(Y)$.

Claim 3.5. $|G: C|=p$. Also, given any $\mu \in \operatorname{Lin}(Y)$ which is an extension of the faithful character $v \in \operatorname{Lin}(Z)$, we have that the stabilizer $G_{\mu}$ of $\mu$ in $G$ is $C$.

Proof. Since $v \in \operatorname{Lin}(Z)$ is a faithful character of the center $Z$ of $G$ and $Y / Z$ is a chief factor of the $p$-group $G$, it follows that the index of the centralizer $C$ of $Y$ in $G$ is $p$.

Claim 3.6. HY $/ Z$ is an elementary abelian p-group. Also, we may assume that $\mathbf{Z}(H Y) \geq Y$ and thus $C=H Y$. 
Proof. Since $|H Y: H|=p$, we have that $(H Y)^{\prime}=\langle[h, k] \mid h, k \in H Y\rangle \leq H$. Observe that $(H Y)^{\prime}$ is normal in $G$ since $H Y$ is normal in $G$ and $(H Y)^{\prime}$ is a characteristic subgroup of $H Y$. Since core ${ }_{G}(H)=Z$, it follows then that $(H Y)^{\prime} \leq Z$. Also, since $Y / Z$ is of order $p$ and $Z<H,(H Y)^{p}=\left\langle k^{p} \mid k \in H Y\right\rangle$ is a characteristic subgroup of the normal subgroup $H Y$ of $G$ and it is contained in $H$. It follows then that $(H Y)^{p} \leq Z$ and thus $H Y / Z$ is an elementary abelian $p$-group.

Observe that the center $\mathbf{Z}(H Y)$ of $H Y$ contains $Z$. If $\mathbf{Z}(H Y)=Z$, then there is a unique character in $\operatorname{Irr}(H)$ lying above $v$ since $H Y / Z$ is an elementary abelian $p$-group and $v \in \operatorname{Lin}(Z)$ is a faithful character, and so $\eta\left(\theta^{G}\right)=1$ or $\eta\left(\theta^{G}\right)=p$, that is a contradiction with (3.1) and therefore it must follow that $\mathbf{Z}(H Y)>Z$. By replacing $Y$ for a normal subgroup of $G$ contained in $\mathbf{Z}(H Y)$ if necessary, we may assume then that $Y \leq \mathbf{Z}(H Y)$ and thus $\mathbf{C}_{G}(Y)=H Y$.

Claim 3.7. The character $\theta \in \operatorname{Irr}(H)$ extends to $H Y=C$. Thus $\theta^{C}$ is the sum of the $p$ distinct extensions of $\theta$.

Proof. Since $|H Y: H|=p$, we have that either $\theta^{H Y} \in \operatorname{Irr}(H Y)$ or $\theta^{H Y}$ is the sum of the $p$ distinct extensions of $\theta$.

Suppose that $\theta^{C} \in \operatorname{Irr}(C)$. Let $\mu \in \operatorname{Lin}(Y)$ be the unique character of $Y$ such that $\left[\left(\theta^{H Y}\right)_{Y}, \mu\right] \neq 0$. Since $G_{\mu}=C$, then $\theta^{G} \in \operatorname{Irr}(G)$. Thus $\theta^{H Y}$ is the sum of the $p$ distinct extensions of $\theta$.

Let $\rho_{1}, \ldots, \rho_{p} \in \operatorname{Irr}(H Y)$ be the $p$ distinct extensions of $\theta$. Since $|G: H Y|=p$, by Lemma 2.2 we must have that

$$
\rho_{i}^{G} \in \operatorname{Irr}(G)
$$

Since $\mathbf{Z}(C) \geq Y$, there is a unique character $\mu_{i} \in \operatorname{Lin}(Y)$ lying below $\rho_{i}$.

Claim 3.9. $\mathbf{Z}(C)=Y$.

Proof. Clearly $Y \leq \mathbf{Z}(C)$. Assume that $Y<\mathbf{Z}(C)$. Let $X \leq \mathbf{Z}(C)$ such that $X / Y$ is a chief factor of $G$ and $Y<X \leq H Y=C$. Observe that such $X$ exists since $H Y$ is normal in $G$, and $X$ is abelian since $X \leq \mathbf{Z}(C)$. We are going to conclude that $v \in \operatorname{Lin}(Z)$ is not a faithful character, which is a contradiction with Claim 3.3.

STEP 3.10. The subgroup $[X, G]$ generates $Y=[X, G] Z$ modulo $Z$.

Proof. Since $Y$ and $X$ are normal subgroups of $G$ with $Y \triangleleft X$ and $|X / Y|=p$, the chief factor $X / Y$ of the $p$-group $G$ is centralized by $G$. So $[X, G] \leq Y$. Suppose that $[X, G] Z<Y$. Since $|Y / Z|=p$, we must have $[X, G] \leq Z=\mathbf{Z}(G)$. So commutation in $G$ induces a bilinear map

$$
d:\left(x Z, g \mathbf{C}_{G}(X)\right) \mapsto[x, g]
$$

of $X / Z \times G / \mathbf{C}_{G}(X)$ into the cyclic group $Z$. This map $d$ is non-singular on the right since $[X, g]=1$ if and only if $g \in \mathbf{C}_{G}(X)$. It is non-singular on the left since $[x, G]=1$ if and only if $x \in Z$. Because $|X: Z|=p^{2}$ and $d$ is a non-singular bilinear form of $X / Z \times$ $G / \mathbf{C}_{G}(X)$ into the cyclic group $Z$, we have $\left|G: \mathbf{C}_{G}(X)\right|=p^{2}$. Since $\lambda \in \operatorname{Lin}(X \mid v)$ extends the faithful character $v \in \operatorname{Irr}(Z)$, this implies that $\mathbf{C}_{G}(X)=G_{\lambda}$. Thus $\mid G$ : $G_{\lambda} \mid=p^{2}$. Since $X \leq \mathbf{Z}(C), C$ fixes $\lambda$. But then $|G: C|=p, C \leq G_{\lambda}$ and $\left|G: G_{\lambda}\right|=p^{2}$. This contradiction proves the claim. 
Given any character $\rho \in \operatorname{Irr}(C)$, since $X \leq \mathbf{Z}(C)$, we have that $\frac{1}{\rho(1)} \rho_{X} \in \operatorname{Lin}(X)$ is the unique character lying below $\rho$.

STEP 3.11. There exist some $\lambda \in \operatorname{Lin}(X)$, some $g \in G \backslash C$ and $i \in\{2, \ldots, p-1\}$ such that $\left[\left(\theta^{C}\right)_{X}, \lambda\right] \neq 0,\left[\left(\theta^{C}\right)_{X}, \lambda^{g}\right] \neq 0$ and $\left[\left(\theta^{C}\right)_{X}, \lambda^{g^{i}}\right] \neq 0$.

Proof. Since $1<\eta\left(\theta^{G}\right)<\frac{p+1}{2}$ and $\rho_{1}^{G}, \ldots, \rho_{p}^{G}$ are the irreducible constituents of $\theta^{G}$, there exist at least 3 distinct $j, k, l \in\{1,2, \ldots, p\}$ such that $\rho_{j}^{G}=\rho_{k}^{G}=\rho_{l}^{G}$. Since $X$ is normal in $G$, by Clifford Theory it follows that $\frac{1}{\rho_{j}(1)}\left(\rho_{j}\right)_{X}, \frac{1}{\rho_{k}(1)}\left(\rho_{k}\right)_{X}$ and $\frac{1}{\rho_{l}(1)}\left(\rho_{l}\right)_{X}$ are $G$-conjugates. Set $\lambda=\frac{1}{\rho_{j}}\left(\rho_{j}\right)_{X}$. Then there exists some $g \in G \backslash C$ such that $\lambda^{g}=\frac{1}{\rho_{k}(1)}\left(\rho_{k}\right)_{X}$. Since $X \leq \mathbf{Z}(C)$ and $|G: C|=p$, there exists some $i \in\{2, \ldots, p-1\}$ such that $(\lambda)^{g^{i}}=\frac{1}{\rho_{l}(1)}\left(\rho_{l}\right)_{X}$.

Fix $g \in G \backslash C$ as in 3.11. Since $X / Y$ is cyclic of order $p, H \cap X>Z$, and $H \cap Y=Z$ we may choose

$$
x \in H \text { such that } X=\langle x, Y\rangle .
$$

Since $X \leq \mathbf{Z}(C)$, we have $[X, C]=1$. Suppose that $\left[x, g^{-1}\right] \in Z$. Then $x$ centralizes both $g^{-1}$ and $C$ modulo $Z$. Hence $x Z \in \mathbf{Z}(G / Z)$, which is false by Step 3.10. Hence $\left[x, g^{-1}\right] \in Y \backslash Z$ and so

$$
Y=Z\langle y\rangle \text { is generated over } Z \text { by } y=\left[x, g^{-1}\right] .
$$

Since $[Y, G] \leq Z$ we have that $z=\left[y, g^{-1}\right] \in Z$. If $z=1$, then $G=C\langle g\rangle$ centralizes $Y=Z\langle y\rangle$, since $C$ centralizes $Y<X$ because $X \leq \mathbf{Z}(C)$, and $G$ centralizes $Z$. This is impossible because $Z=\mathbf{Z}(G)<Y$. Thus

$$
z=\left[y, g^{-1}\right] \text { is a non-trivial element of } Z .
$$

By (3.13) we have $y=\left[x, g^{-1}\right]=x^{-1} x^{g^{-1}}$. By (3.14) we have $z=\left[y, g^{-1}\right]=y^{-1} y^{g^{-1}}$. Finally $z^{g^{-1}}=z$ since $z \in Z$. Since $X=Z\langle x, y\rangle$ is abelian since $X \leq \mathbf{Z}(C)$, it follows that

$$
z^{g^{-j}}=z, y^{g^{-j}}=y z^{j} \text { and } x^{g^{-j}}=x y^{j} z^{\left(\begin{array}{l}
j \\
2
\end{array}\right)},
$$

for any integer $j=0,1, \ldots, p-1$. Because $g^{-p} \in C$ centralizes $X$ since $X \leq \mathbf{Z}(C)$, we have

$$
z^{p}=1 \text { and } y^{p} z^{\left(\begin{array}{l}
p \\
2
\end{array}\right)}=1
$$

Since $p>2$ is odd by hypothesis, $p$ divides $\left(\begin{array}{l}p \\ 2\end{array}\right)=\frac{p(p-1)}{2}$ and $z^{\left(\begin{array}{c}p \\ 2\end{array}\right)}=1$. Therefore $y^{p}=$ $z^{p}=1$. It follows that $y^{i}, z^{i}$ and $z^{\left(\begin{array}{c}i \\ 2\end{array}\right)}$ depend only on the residue of $i$ modulo $p$, for any integer $i \geq 0$. such that $X=Y\langle x\rangle$ and $x \in C$. Thus by (3.14) we have that

$$
z^{\left(\begin{array}{l}
j \\
2
\end{array}\right)} \neq 1 \text { for any integer } 0<j<p .
$$

Let $\lambda \in \operatorname{Lin}(X)$ and $i \in\{2, \ldots, p-1\}$ be as in Step 3.11. Set $\varpi=\frac{1}{\theta(1)} \theta_{X \cap H}$. We can check that $\varpi \in \operatorname{Lin}(X \cap H)$. Since $\left(\theta^{C}\right)_{X}=\left(\theta_{H \cap X}\right)^{X}$, we have that $\lambda, \lambda^{g}$ and $\lambda^{g^{i}}$ are 
extensions of $\varpi$. Since $x \in(H \cap X)$, by the previous statement we have that

$$
\lambda(x)=\lambda^{g}(x)=\lambda^{g^{i}}(x) .
$$

By (3.15) we have that

$$
\lambda^{g}(x)=\lambda\left(x^{g^{-1}}\right)=\lambda(x y)=\lambda(x) \lambda(y) .
$$

Thus by (3.17), we get

$$
\lambda(y)=1 .
$$

Therefore

$$
\begin{aligned}
\lambda^{g^{i}}(x) & =\lambda\left(x^{g^{-i}}\right) \\
& =\lambda\left(x y^{i} z^{\left(\begin{array}{c}
i \\
2
\end{array}\right)}\right) \quad \text { by }(3.15) \\
& =\lambda(x) \lambda\left(y^{i}\right) \lambda\left(z^{\left(\begin{array}{l}
i \\
2
\end{array}\right)}\right) \\
& =\lambda(x) \lambda\left(z^{\left(\begin{array}{c}
i \\
2
\end{array}\right)}\right),
\end{aligned}
$$

where the last line follows from (3.18). By (3.17), we have that $\lambda\left(z^{\left(\begin{array}{l}2 \\ 2\end{array}\right)}\right)=1$. But $\lambda_{Z}=$ $v \in \operatorname{Lin}(Z)$ is a faithful character and $z^{(i)} \neq 1$ by (3.16). This is a contradiction and the claim is proved.

Since $\mathbf{Z}(H Y)=Y$, we have that $\mathbf{Z}(H)=Z$. Thus $H Y$ is a class 2 group with $H Y / Z$ elementary abelian. Therefore $\theta \in \operatorname{Irr}(H)$ is the only character in $H$ lying above $v \in \operatorname{Lin}(Z)$. Hence an irreducible character of $G$ lies over $\theta$ if and only if it lies over $v$. Since $\operatorname{Irr}(G \mid v)$ has either 1 element or at least $p$ by Lemma 2.2, it follows that $\eta\left(v^{G}\right)=1$ or $\eta\left(v^{G}\right) \geq p$, and therefore either $\eta\left(\theta^{G}\right)=1$ or $\eta\left(\theta^{G}\right) \geq p$. But $1<\eta\left(\theta^{G}\right)<\frac{p+1}{2}$, and that is our final contradiction and thus the statement of Theorem A holds.

4. Examples. In this section, we will prove that the group $G$, the subgroup $H$ and the character $\lambda \in \operatorname{Lin}(H)$ that satisfy Hypothesis 4.1 have the properties that $|G: H|=p^{2}$ and $\eta\left(\lambda^{G}\right)=\frac{p+1}{2}$. And then, given any integer $n \geq 2$, we construct a group $G$ with a subgroup $H$ and a character $\lambda \in \operatorname{Lin}(H)$ such that $|G: H|=p^{n}$ and $\eta\left(\lambda^{G}\right)=\frac{p+1}{2}$.

HYPOTHESIS 4.1. Fix an odd prime $p$. Let $G$ be the semidirect product of a cyclic group $C$ of order $p$ and an elementary abelian group $A$ of order $p^{3}$. Assume $C=\langle c\rangle$ and

$$
A=\langle a\rangle \times\langle[a, c]\rangle \times\langle[a, c, c]\rangle,
$$

for some a in A. Observe that the subgroup $\{e\} \times\{e\} \times\langle[a, c, c]\rangle$ is the center of the group G. Set $Z=\{e\} \times\{e\} \times\langle[a, c, c]\rangle$.

Fix $\omega$ a primitive complex $p$-th root of unity. Let $\alpha \in \operatorname{Lin}(\langle a\rangle), \beta \in \operatorname{Lin}(\langle[a, c]\rangle)$ and $\gamma \in \operatorname{Lin}(\langle[a, c, c]\rangle)$ be the unique linear characters such that $\alpha(a)=\beta([a, c])=$ $\gamma([a, c, c])=\omega$.

Set

$$
H=\langle a\rangle \times\{e\} \times\langle[a, c, c]\rangle \text { and } \lambda=1_{\langle a\rangle} \times 1_{\{e\}} \times \gamma \in \operatorname{Lin}(H) .
$$


Observe that $H$ is a subgroup of $A$ of index $p$. Thus $|G: H|=p^{2}$. Observe also that $\lambda$ extends to $A$ and there are exactly $p$ distinct extensions of $\lambda$ to $A$, namely

$$
\operatorname{Irr}(A \mid \lambda)=\left\{1_{\langle a\rangle} \times \beta^{r} \times \gamma \mid r=0,1, \ldots, p-1\right\} .
$$

Set $\Lambda_{r}=1_{\langle a\rangle} \times \beta^{r} \times \gamma$.

LEMma 4.5. Assume Hypothesis 4.1. Given any integer $i$ with $0<i$, we have that

$$
\left(\Lambda_{r}\right)^{c^{i}}=\alpha^{r i+\frac{i(i-1)}{2}} \times \beta^{r+i} \times \gamma .
$$

Proof. Observe that $\left(\Lambda_{r}\right)^{c}=\alpha^{r} \times \beta^{r} \beta \times \gamma=\alpha^{r} \times \beta^{r+1} \times \gamma$ since $a^{c}=a[a, c]$ and $[a, c]^{c}=[a, c][a, c, c]$. Assume by induction that

$$
\left(\Lambda_{r}\right)^{c^{n}}=\alpha^{r n+\frac{n(n-1)}{2}} \times \beta^{r+n} \times \gamma .
$$

Then

$$
\begin{aligned}
\left(\Lambda_{r}\right)^{c^{n+1}} & =\left(\left(\Lambda_{r}\right)^{c^{n}}\right)^{c} \\
& =\left(\alpha^{r n+\frac{n(n-1)}{2}} \times \beta^{r+n} \times \gamma\right)^{c} \text { by }(4.6) \\
& =\alpha^{r n+\frac{n(n-1)}{2}+r+n} \times \beta^{r+n+1} \times \gamma,
\end{aligned}
$$

where the last line follows since $a^{c}=a[a, c]$ and $[a, c]^{c}=[a, c][a, c, c]$. We can check that $r n+\frac{n(n-1)}{2}+r+n=r(n+1)+\frac{(n+1)(n)}{2}$. Thus

$$
\left(\Lambda_{r}\right)^{c^{n+1}}=\alpha^{r(n+1)+\frac{(n+1) n}{2}} \times \beta^{r+(n+1)} \times \gamma,
$$

and the result follows by induction.

Lemma 4.7. Assume Hypothesis 4.1. Let $r$ be an integer such that $0<r<p$. Then $\left(\Lambda_{r}\right)^{c^{j}}$ is an extension of $\lambda$ if and only if either $j \equiv 0 \bmod p$ or $j \equiv(1-2 r) \bmod p$. If $i \equiv(1-2 r) \bmod p$ then $\left(\Lambda_{r}\right)^{c^{i}}=\Lambda_{1-r}$.

Proof. By Lemma 4.5, we have that $\left(\Lambda_{r}\right)^{c^{i}}$ is an extension of $\lambda$ if and only if $\alpha^{i r+\frac{i(i-1)}{2}}=1_{\langle a\rangle}$. Since $\alpha$ is a faithful linear character of a cyclic group of order $p, \alpha^{i r+\frac{i(i-1)}{2}}=1_{\langle a\rangle}$ if and only if $\left(i r+\frac{i(i-1)}{2}\right) \equiv 0 \bmod p$. Observe that $\left(i r+\frac{i(i-1)}{2}\right) \equiv$ $0 \bmod p$ if and only if either $i \equiv 0 \bmod p$ or $\left(r+\frac{i-1}{2}\right) \equiv 0 \bmod p$. Therefore $\left(\Lambda_{r}\right)^{c^{i}}$ is an extension of $\lambda$ if and only if either $i \equiv 0 \bmod p$ or $i \equiv(1-2 r) \bmod p$.

If $i \equiv(1-2 r) \bmod p$, then $\left(\Lambda_{r}\right)^{c^{i}}=\Lambda_{1-r}$ by Lemma 4.5.

Lemma 4.8. Assume Hypothesis 4.1. Then $1<\eta\left(\lambda^{G}\right) \leq \frac{p+1}{2}$.

Proof. By the previous lemma, it follows that the stabilizer of $\Lambda_{r}$ is a proper subgroup of $G$. Since $|G: A|=p$ and $\Lambda_{r} \in \operatorname{Lin}(A)$, we have that

$$
\left(\Lambda_{r}\right)^{G} \in \operatorname{Irr}(G) \text { for any integer } r \text {. }
$$

Since $p>2$, it follows that there exist two distinct integers $k, l$ such that $0<k, l<p$ and $k \neq(1-2 l) \bmod p$. Thus by Lemma 4.7 we have that $\Lambda_{k}$ and $\Lambda_{l}$ are not 
$G$-conjugates. It follows that $\left(\Lambda_{k}\right)^{G} \neq\left(\Lambda_{l}\right)^{G}$. Since $\left(\Lambda_{k}\right)^{G} \neq\left(\Lambda_{l}\right)^{G},\left(\Lambda_{k}\right)^{G},\left(\Lambda_{l}\right)^{G} \in$ $\operatorname{Irr}(G)$ and both $\Lambda_{k}$ and $\Lambda_{l}$ lie above $\lambda$, we have that $\eta\left(\lambda^{G}\right) \geq 2$.

Observe that $r \equiv(1-r) \bmod p$ if and only if $2 r \equiv 1 \bmod p$. Thus given any $r$ such that $0<r<p$ and $2 r \neq 1 \bmod p$, by Lemma 4.7 we have that $\Lambda_{r}, \Lambda_{1-r} \in \operatorname{Irr}(A)$ are two distinct $G$-conjugate extensions of $\lambda$. Thus $\eta\left(\lambda^{G}\right) \leq \frac{p+1}{2}$.

Proposition 4.10. Assume Hypothesis 4.1. Then $|G: H|=p^{2}$ and $\eta\left(\lambda^{G}\right)=\frac{p+1}{2}$.

Proof. By Lemma 4.8, we have that $1<\eta\left(\lambda^{G}\right) \leq \frac{p+1}{2}$. Thus by Theorem A, it follows that $\eta\left(\lambda^{G}\right)=\frac{p+1}{2}$.

Denote by $1_{H}$ the principal character of $H$.

LEMMA 4.11. Let $p$ be a prime number, $G$ be a p-group and $H$ be a subgroup of $G$ with $|G: H|=p^{n}$. Then $\eta\left(\left(1_{H}\right)^{G}\right) \geq n(p-1)+1$.

Proof. We are going to use a double induction, first on $|G|$ and then on $n$, where $|G: H|=p^{n}$. Using induction on the order of $G$, without lost of generality we may assume that $\operatorname{core}_{G}(H)=1$.

Let $Z_{1}$ be a subgroup of the center $\mathbf{Z}(G)$ of $G$ with $\left|Z_{1}\right|=p$. Observe that $H \cap Z_{1}=$ 1 since $\operatorname{core}_{G}(H)=1$. Thus $\left|H Z_{1}: H\right|=p$. By Lemma 2.3, we have that

$$
\eta\left(\left(1_{H}\right)^{G}\right) \geq \eta\left(\left(1_{H Z_{1}}\right)^{G}\right)+(p-1) .
$$

Since $\left|G: H Z_{1}\right|=p^{n-1}$, by induction on $n$ we have that

$$
\eta\left(\left(1_{H Z_{1}}\right)^{G}\right) \geq(n-1)(p-1)+1 .
$$

The result follows by (4.12) and the previous statement.

LeMma 4.13. Let $G_{0}$ be a p-group and $\Gamma$ be a character of $G_{0}$. Assume that $\left[\Gamma, 1_{G_{0}}\right]=$ 0 . Let $N=G_{0} \times G_{0} \times \cdots \times G_{0}$ be the direct product of $p$-copies of $G_{0}$. Set

$$
\Delta=\Gamma \times 1_{G_{0}} \times \cdots \times 1_{G_{0}} .
$$

Let $C=\langle c\rangle$ be a cyclic group of order $p$. Observe that $C$ acts on $N$ by

$$
c:\left(n_{0}, n_{1}, \ldots, n_{p-1}\right) \mapsto\left(n_{p-1}, n_{0}, \ldots, n_{p-2}\right)
$$

for any $\left(n_{0}, n_{1}, \ldots, n_{p-1}\right) \in N$.

Let $G$ be the direct product of $N$ and $C$, i.e, $G$ is the wreath product of $G_{0}$ and $C$. Then $\eta\left(\Delta^{G}\right)=\eta(\Gamma)$.

Proof. Let $\delta \in \operatorname{Irr}(N)$ be a constituent of $\Delta$. Observe that $\delta$ is of the form $\gamma \times$ $1_{G_{0}} \times \cdots \times 1_{G_{0}}$, for some $\gamma \in \operatorname{Irr}\left(G_{0}\right)$ such that $[\gamma, \Gamma] \neq 0$. Observe that $\gamma \neq 1_{G_{0}}$ since $\left[\Gamma, 1_{G_{0}}\right]=0$. By (4.14), we have that $\delta$ is $G$-invariant if and only if $\gamma=1_{G_{0}}$. Thus $\delta^{G} \in \operatorname{Irr}(G)$ for any constituent $\delta \in \operatorname{Irr}(N)$ of $\Delta$. Observe that the $G$-orbit of $\delta \in \operatorname{Irr}(N)$ is

$$
\left\{\gamma \times 1_{G_{0}} \times \cdots \times 1_{G_{0}}, 1_{G_{0}} \times \gamma \times \cdots \times 1_{G_{0}}, \cdots, 1_{G_{0}} \times \ldots \times 1_{G_{0}} \times \gamma\right\} .
$$


Thus if $\delta, \epsilon \in \operatorname{Irr}(N)$ are two distinct constituents of $\Delta$, then $\delta^{G} \neq \epsilon^{G}$. It follows that $\eta\left(\Delta^{G}\right)=\eta(\Gamma)$.

THEOREM 4.15. Let $p$ be an odd prime number and $n \geq 2$ be an integer. There exist a p-group $G$, a subgroup $H$ of $G$ and $\lambda \in \operatorname{Lin}(H)$, such that $|G: H|=p^{n}$ and $\eta\left(\lambda^{G}\right)=\frac{p+1}{2}$.

Proof. If $n=2$, then the result follows by Lemma 4.10. By induction on $n$, we may assume that the result holds for any integer $n$ such that $n-1 \geq 2$.

Fix a $p$-group $G_{0}$, a subgroup $H_{0} \leq G_{0}$ and $\lambda_{0} \in \operatorname{Lin}\left(H_{0}\right)$ such that:

$$
\left|G_{0}: H_{0}\right|=p^{n-1} \text { and } \eta\left(\lambda_{0}^{G_{0}}\right)=\frac{p+1}{2} .
$$

Let $N$ and $G$ be as in Lemma 4.13. Let

$$
H=H_{0} \times G_{0} \times \ldots \times G_{0} .
$$

Then $H$ is a subgroup of $N$ and $|G: H|=|G: N|\left|N: H_{0}\right|=p\left|G_{0}: H_{0}\right|=p^{n}$.

Set $\lambda=\lambda_{0} \times 1_{G_{0}} \times \ldots \times 1_{G_{0}}$. Observe that $\lambda \in \operatorname{Lin}(H)$ since $\lambda_{0} \in \operatorname{Lin}\left(H_{0}\right)$. We can check that $\eta\left(\lambda^{N}\right)=\eta\left(\lambda_{0}^{G_{0}}\right)$. Thus by (4.16) we have that $\eta\left(\lambda^{N}\right)=\frac{p+1}{2}$.

By Lemma 4.11, we have that $\lambda_{0} \neq 1_{H_{0}}$. Thus $\left[\lambda_{0}^{G_{0}}, 1_{G_{0}}\right]=0$. By Lemma 4.13 we have then that $\eta\left(\lambda^{N}\right)=\eta\left(\lambda^{G}\right)$ and the result is proved.

LEMMA 4.17. Let $p$ be a prime number such that $p-1$ is divisible by 3 . Fix $r \in$ $\{1, \ldots, p-1\}$. Then the set $\left\{r\left(1-i^{3}\right) \bmod p \mid i=0, \ldots, p-1\right\}$ has $\frac{p+2}{3}$ elements. Also, given any $e \in\left\{r\left(1-i^{3}\right) \bmod p \mid i=1, \ldots, p-1\right\}$, there are exactly 3 distinct solutions in $\{1, \ldots, p-1\}$ of the equation $e \equiv r\left(1-x^{3}\right) \bmod p$

Proof. Let $u$ be a generator of the units of the field $F$ of $p$ elements. Then $U=\left\langle u^{\frac{p-1}{3}}\right\rangle$ is a subgroup of order 3 and any element in $U$ is a solution of $x^{3} \equiv 1 \bmod p$. Thus given any integer $n \neq r$, if the equation $x^{3} \equiv r-n \bmod p$ has a solution, then it has exactly 3 distinct solutions in $F$. Therefore the set $\left\{r\left(1-i^{3}\right) \bmod p \mid i=1, \ldots, p-1\right\}$ has $\frac{p-1}{3}$ distinct elements. Since $0^{3}=0$, the set $\left\{\left(r\left(1-i^{3}\right) \bmod p \mid i=0, \ldots, p-1\right\}\right.$ has $\frac{p-1}{3}+1=\frac{p+2}{3}$ elements.

HYPOTHESIS 4.18. Let $p>5$ be a prime number such that $p-1$ is divisible by 3 . Let $F$ be a field of $p$ elements and $F[x]$ be the truncated polynomial algebra generated over $F$ by some $x$ satisfying only $x^{4}=0$. So $F[x]$ is a vector space of dimension 4 over $F$ with $1, x, x^{2}$ and $x^{3}$ as a basis. Let $m$ be an isomorphism of the additive group $F[x]^{+}$of $F[x]$ onto a multiplicative group $M$. Then $M$ is an elementary abelian multiplicative group of order $p^{4}$ with $m(1), m(x), m\left(x^{2}\right), m\left(x^{3}\right)$ as generators. Let $U$ be the subgroup of the unit group $F[x]^{\times}$generated by $1+x$ and $1+x^{2}$. The general element of $U$ is

$$
(1+x)^{i}\left(1+x^{2}\right)^{j}=1+i x+\left(\left(\begin{array}{l}
i \\
2
\end{array}\right)+j\right) x^{2}+\left(\left(\begin{array}{l}
i \\
3
\end{array}\right)+i j\right) x^{3}
$$

for arbitrary integers $i, j$, since $x^{4}=0$. Because $p>3$, it follows that $U$ is elementary abelian of order $p^{2}$, and that (4.19) holds for any $i, j \in F$. The group $U$ acts naturally on the group $M$, so that

$$
m(y)^{u}=m(y u)
$$


for all $y \in F[x]$ and $u \in U$. Let $G$ be the semidirect product of $M$ and $U$. Then $G$ is a multiplicative group with order $p^{6}$.

Let $H$ be the subgroup

$$
H=\left\langle m(1), m(x), m\left(x^{3}\right)\right\rangle=\left\{m\left(a_{0}+a_{1} x+a_{3} x^{3}\right) \mid a_{0}, a_{1}, a_{3} \in F\right\} .
$$

Fix a primitive $p$-th root of unity $\omega$. Fix an integer $r>0$ such that $3 r \equiv-1 \bmod p$. Thus $r \equiv \frac{-1}{3} \bmod p$ and $r \not \equiv 0 \bmod p$. Let $\lambda \in \operatorname{Lin}(H)$ be the character given by

$$
\lambda\left(m\left(a_{0}+a_{1} x+a_{3} x^{3}\right)\right)=\omega^{r a_{0}+r a_{1}+a_{3}} .
$$

TheOrem 4.23. Assume Hypothesis 4.18. Then

$$
\lambda^{G}=\chi_{0}+3 \sum_{i=1}^{\frac{p-1}{3}} \chi_{i}
$$

where $\chi_{i} \in \operatorname{Irr}(G)$ and $\chi_{i} \neq \chi_{j}$ if $i \neq j$ for $i, j=0,1, \ldots, \frac{p-1}{3}$. Thus $\eta(\lambda)=\frac{p+2}{3}$.

Proof. The center $\mathbf{Z}(G)$ of $G$ is the subgroup $\left\langle m\left(x^{3}\right)\right\rangle$ of order $p$. Let $\gamma$ be the faithful linear character of $\mathbf{Z}(G)$ sending $m\left(x^{3}\right)$ to $\omega$. Then $\operatorname{Lin}(M \mid \gamma)$ consists of the $p^{3}$ linear characters $\mu_{f_{0}, f_{1}, f_{2}}$, for $f_{0}, f_{1}, f_{2} \in F$ given by

$$
\mu_{f_{0}, f_{1}, f_{2}}\left(m\left(a_{0}+a_{1} x+a_{2} x^{2}+a_{3} x^{3}\right)\right)=\omega^{f_{0} a_{0}+f_{1} a_{1}+f_{2} a_{2}+a_{3}}
$$

for all $a_{0}, a_{1}, a_{2}, a_{3} \in F$. If $e, i, j \in F$, then (4.19) and (4.20) imply that the conjugate character $\mu_{e, 0,0}^{(1+x)^{-i}\left(1+x^{2}\right)^{-j}}$ to $\mu_{e, 0,0}$ sends

$$
\begin{aligned}
& m(1) \mapsto \mu_{e, 0,0}\left(m\left(1+i x+\left(\left(\begin{array}{l}
i \\
2
\end{array}\right)+j\right) x^{2}+\left(\left(\begin{array}{l}
i \\
3
\end{array}\right)+i j\right) x^{3}\right)\right)=\omega^{e+\left(\begin{array}{l}
i \\
3
\end{array}\right)+i j}, \\
& m(x) \mapsto \mu_{e, 0,0}\left(m\left(x+i x^{2}+\left(\left(\begin{array}{l}
i \\
2
\end{array}\right)+j\right) x^{3}\right)\right)=\omega^{\left(\begin{array}{c}
i \\
2
\end{array}\right)+j}, \\
& m\left(x^{2}\right) \mapsto \mu_{e, 0,0}\left(m\left(x^{2}+i x^{3}\right)\right)=\omega^{i}, \\
& m\left(x^{3}\right) \mapsto \mu_{e, 0,0}\left(m\left(x^{3}\right)\right)=\omega .
\end{aligned}
$$

It follows that

$$
\mu_{e, 0,0}^{(1+x)^{-i}\left(1+x^{2}\right)^{-j}}=\mu_{e+\left(\begin{array}{c}
i \\
3
\end{array}\right)+i j,\left(\begin{array}{l}
i \\
2
\end{array}\right)+j, i}
$$

for any $e, i, j \in F$. If we fix $e$, then the above equation implies that distinct pairs $(i, j) \in F \times F$ yield distinct conjugates $\mu_{e, 0,0}^{(1+x)^{-i}\left(1+x^{2}\right)^{-j}} \in \operatorname{Lin}(M \mid \gamma)$. Hence the $G$-orbit $L_{e}$ of $\mu_{e, 0,0}$ has exactly $p^{2}$ members. Furthermore the above equation implies that the only member of that orbit with the form $\mu_{f, 0,0}$ is $\mu_{e, 0,0}$. We conclude that the orbits 
$L_{e}$, for $e \in F$, are $p$ distinct $G$-orbits in $\operatorname{Lin}(M \mid \gamma)$, each with size $p^{2}$. Since the normal subgroup $M$ of index $p^{2}$ is exactly the stabilizer of $\mu_{e, 0,0} \in \operatorname{Lin}(M)$ in $G$, the induced characters

$$
\chi_{e}=\mu_{e, 0,0}^{G} \text { are precisely the distinct members of } \operatorname{Irr}(G \mid \gamma)
$$

Then

$$
\lambda^{M}=\sum_{f \in F} \mu_{r, r, f} \text { and } \lambda^{G}=\sum_{f \in F} \mu_{r, r, f}^{G}
$$

Claim 4.29. Let $i \in\{1, \ldots, p-1\}, e=r\left(1-i^{3}\right)$ and $j=r-\left(\begin{array}{l}i \\ 2\end{array}\right)$. Then

$$
\mu_{e, 0,0}^{(1+x)^{-i}\left(1+x^{2}\right)^{-j}}=\mu_{r, r, i}
$$

Proof. For a fixed $i$, we have

$$
\begin{aligned}
e+\left(\begin{array}{l}
i \\
3
\end{array}\right)+i j & =e+\left(\begin{array}{l}
i \\
3
\end{array}\right)+i\left(r-\left(\begin{array}{l}
i \\
2
\end{array}\right)\right) \\
& =e+\frac{i(i-1)(i-2)}{6}+i\left(r-\frac{i(i-1)}{2}\right) \\
& =i^{3}\left(\frac{1}{6}-\frac{1}{2}\right)+i^{2}\left(\frac{1}{2}-\frac{1}{2}\right)+i\left(r+\frac{1}{3}\right)+e \\
& \equiv \frac{-i^{3}}{3}+e \bmod p, \quad \text { since } r \equiv \frac{-1}{3} \bmod p \\
& \equiv \frac{-i^{3}}{3}+r\left(1-i^{3}\right) \bmod p, \quad \text { since } e=r\left(1-i^{3}\right) \\
& \equiv r-i^{3}\left(r+\frac{1}{3}\right) \equiv r \bmod p,
\end{aligned}
$$

where the last line follows since $r \equiv \frac{-1}{3} \bmod p$. Thus $\left(e+\left(\begin{array}{l}i \\ 3\end{array}\right)+i j,\left(\begin{array}{l}i \\ 2\end{array}\right)+j, i\right)=(r, r, i)$ in $F \times F \times F$ and so by (4.26) we get (4.30).

By the previous claim and (4.28), we have that

$$
\lambda^{G}=\sum_{i=0}^{p-1} \mu_{r\left(1-i^{3}\right), 0,0}^{G} .
$$

By Lemma 4.17, we have then

$$
\lambda^{G}=\mu_{r, 0,0}^{G}+3 \sum_{e \in\left\{r\left(1-i^{3}\right) \mid i=1, \ldots, p-1\right\}} \mu_{e, 0,0}^{G} .
$$

By (4.27) we have that $\mu_{e, 0,0}^{G} \in \operatorname{Irr}(G)$ and $\mu_{e, 0,0}^{G} \neq \mu_{f, 0,0}^{G}$ if $e \not \equiv f \bmod p$. Thus by Lemma 4.17 and (4.31), we conclude that $\eta\left(\lambda^{G}\right)=\frac{p+2}{3}$ and the proof is complete. 
Acknowledgment. Professor Everett C. Dade brought to my attention Proposition 4.10, the main step in the proof of Theorem 4.15, and Lemma 4.11. The proofs of those are based on his arguments, which are included here with his permission. I thank him for this and for very useful conversations and emails. I also thank the referee of a previous version of this note for helpful comments and suggestions and for providing Theorem 4.23 when $p=7$, which is included here along with the suggestions with her/his permission.

\section{REFERENCES}

1. E. Adan-Bante, Products of characters and finite p-groups, J. Algebra 277 (1) (2004), $236-255$

2. I. M. Isaacs Character theory of finite groups (Academic Press, 1976). 